\title{
Extension of the dielectric breakdown model for simulation of viscous fingering at finite viscosity ratios
}

\author{
Shashvat Doorwar and Kishore K. Mohanty \\ Department of Petroleum and Geosystems Engineering, University of Texas, Austin, Texas 78712, USA \\ (Received 12 March 2014; revised manuscript received 25 May 2014; published 30 July 2014)
}

\begin{abstract}
Immiscible displacement of viscous oil by water in a petroleum reservoir is often hydrodynamically unstable. Due to similarities between the physics of dielectric breakdown and immiscible flow in porous media, we extend the existing dielectric breakdown model to simulate viscous fingering patterns for a wide range of viscosity ratios $\left(\mu_{r}\right)$. At low values of power-law index $\eta$, the system behaves like a stable Eden growth model and as the value of $\eta$ is increased to unity, diffusion limited aggregation-like fractals appear. This model is compared with our two-dimensional (2D) experiments to develop a correlation between the viscosity ratio and the power index, i.e., $\eta=10^{-5} \mu_{r}{ }^{0.8775}$. The 2D and three-dimensional (3D) simulation data appear scalable. The fingering pattern in 3D simulations at finite viscosity ratios appear qualitatively similar to the few experimental results published in the literature.
\end{abstract}

DOI: 10.1103/PhysRevE.90.013028

PACS number(s): 47.53.+n, 47.20.-k, 47.54.-r, 47.56.+r

\section{INTRODUCTION}

Local pore-scale fluctuations and variations in fluid flow in porous media constantly excite the instability of two-phase immiscible displacements. For a stable displacement, these pore-scale instabilities fade out and a stable macroscopic diffused front is observed. Alternately, at adverse viscosity ratios ( $\left.\mu_{r}=\mu_{\text {displaced }} / \mu_{\text {injected }}\right)$ or in the presence of large-scale heterogeneities, these fluctuations may grow into macroscale fingers, as reported in several laboratory and reservoir-scale floods [1].

Modeling such unstable flows has always been a challenge, partly due to the numerical dispersion error associated with the conventional continuum based simulation techniques and due to the fact that Darcy's law represents only the average flux. A similar inadequacy in the Richard's equation has been pointed out by Cueto-Felgueroso and Juanes [2]. They introduced local and nonlocal energy terms in the Richard's equation to better predict the channeling of water in unsaturated porous media. Others have suggested stochastic or probabilistic methods for simulation of viscous fingers [3,4]. Diffusion limited aggregation (DLA) was introduced by Witten and Sanders [5] as a random walk model to describe formation of aggregates in nature. The model produced structures that resemble viscous fingers in the limit of infinite viscosity ratio and were studied in detail by Patterson [6] and Lenormand [1]. Many have investigated the effect of the stickiness factor in DLA to make the structure of the fingers qualitatively different: crossover from dense to DLA fractals [7-10]. The width of the fingers increases as the stickiness factor is increased, but even for high stickiness factor the model fails to predict the displacement patterns expected at finite viscosity ratios.

Similar to DLA, the dielectric breakdown model (DBM) was introduced for the modeling of dielectric discharge in insulators by Niemeyer et al. [11] and later explained at the molecular level by Pietronero and Wiesmann [12]. Due to conceptual and qualitative similarity between DLA, DBM, and viscous fingering, DBM was also used as an analogy to unstable flows in porous media $[13,14]$. Indeed, the flow in porous media has similarities with the dielectric breakdown and good understanding of the process may lead to a better modeling of viscous instabilities. Recently, Clemens et al. [15] modeled similar viscous instabilities using Computational Fluid Dynamics (CFD) simulation. CFD simulations can also be a good tool to model such viscous instabilities, but they tend to be computationally intensive and can be applied only in a small domain. The goal here is to develop a simplified model for viscous fingering which can be used later for scale-up.

In this study, the similarity between dielectric breakdown and viscous fingering is revisited and the existing DBM is modified to develop a pore-scale network model (in both two and three dimensions) that is capable of predicting viscous fingering for finite viscosity ratios. At low viscosity ratios, a stable, uniform Buckley-Leverett type displacement front is obtained. At adverse, yet finite, viscosity ratios instabilities arise and grow with time. At very adverse viscosity ratio limits, the model produces DLA type fractal fingers. A comparison between the results of two-dimensional (2D) and threedimensional (3D) computational simulation and micromodel experiments has been discussed for various viscosity ratios and a correlation has been proposed.

\section{MODEL}

There are multiple similarities between dielectric breakdown and flow in porous media. Viscous fingers at high viscosity ratios display a fractal nature-like dielectric breakdown. Both processes are examples of particles flowing from high potentials to low potentials along the path of least resistance. Dielectric breakdown can be considered as a specific case of viscous fingering where high mobility particles (electrons) are discharged into a medium that offers infinite resistivity (air). Dielectric discharge occurs only above a certain potential called the critical excitation potential $\left(E_{c}\right)$ and a nonwetting phase also enters a pore throat above a critical capillary pressure $\left(P_{c}\right)$. Both viscous fingers and dielectric discharge have a higher growth probability at the tip [11]. When a viscous finger grows, there is a region (typically between two adjacent fingers) around where new fingers cannot grow, just like a region screened by a conductor in an electric field, known as Faraday's screening [11]. 
Niemeyer et al. [11] presented a two-dimensional stochastic model to describe the discharge patterns of dielectric breakdown with a basic assumption that the growth probability depends on the local electric field. In this model, the domain is approximated by a lattice (consisting of sites and bonds); the central site is assumed as one of the electrodes and the other electrode is modeled as a circle at a large enough distance, so the pattern grows radially outwards. The discharge pattern grows in a stepwise manner where electric potential is defined for each site in the lattice by a discrete Laplace equation with the boundary condition $\phi=0$ at each site of the discharge pattern and $\phi=1$ at the external circle. Thus

$$
\nabla^{2} \phi=0 .
$$
2D:

The discrete forms of Eq. (1) can be written as

$$
\phi_{i, j}=\frac{1}{4}\left(\phi_{i-1, j}+\phi_{i+1, j}+\phi_{i, j-1}+\phi_{i, j+1}\right),
$$

3D:

$$
\begin{aligned}
\phi_{i, j, k}= & \frac{1}{6}\left(\phi_{i-1, j, k}+\phi_{i+1, j, k}+\phi_{i, j-1, k}+\phi_{i, j+1, k}\right. \\
& \left.+\phi_{i, j, k-1}+\phi_{i, j, k+1}\right) .
\end{aligned}
$$

The discharge pattern occupies a part of the lattice. At each step, one site is added adjacent to an occupied site on the discharge pattern depending on its probability. A probability is associated with the transport of electrons from an occupied site $(i j)$ at the edge of the swept region to each adjacent site $\left(i^{\prime} j^{\prime}\right)$ which is a function of the local electric field, i.e.,

$$
P\left(i j \rightarrow i^{\prime} j^{\prime}\right)=\frac{\left(\phi_{i^{\prime} j^{\prime}}\right)^{\eta}}{\sum\left(\phi_{i^{\prime} j^{\prime}}\right)^{\eta}},
$$

where $\eta$ is a parameter of the system. This probability is multiplied by a random number between 0 and 1 to introduce a randomness to the system. The site with the maximum probability is chosen as the new invaded site.

With the new site added to the discharge pattern, the new resultant electric field is recomputed in the uninvaded region. This procedure is repeated until the discharge pattern hits the external electrode. Niemeyer et al. [11] concentrated on a particular case with $\eta=1$ (i.e., growth probability proportional to local field). The computed patterns matched their experimental discharge (Lichtenberg figures) on a 2-mm glass plate in $0.3 \mathrm{MPa} \mathrm{SF}_{6}$. In the same study, they also pointed out that for systems other than gases (solids, liquids, and polymers) the microscopic relation between the growth probability and local field may be more appropriately described by a nonlinear function. For $\eta=0$, the pattern became like the Eden model, which grows homogeneously and has integral fractal dimension $D=2$ [16]. This observation is of interest because in two-phase immiscible displacements in porous media, displacement fronts change from being stable $(D=2)$ at low viscosity ratios $\left(\mu_{r}<1\right)$ to unstable and fractal-like at very high viscosity ratios, as shown by Doorwar and Mohanty [17].

Pietronero et al. [12] provided a microscopic interpretation of the various elements of Niemeyer's dielectric breakdown model. They explained the origin of the stochastic nature and the power-law dependence of the model through ionization of gas molecules and propagation of electrons [Fig. 1(a)]. They describe that the process of discharge propagation requires (a)

(b)
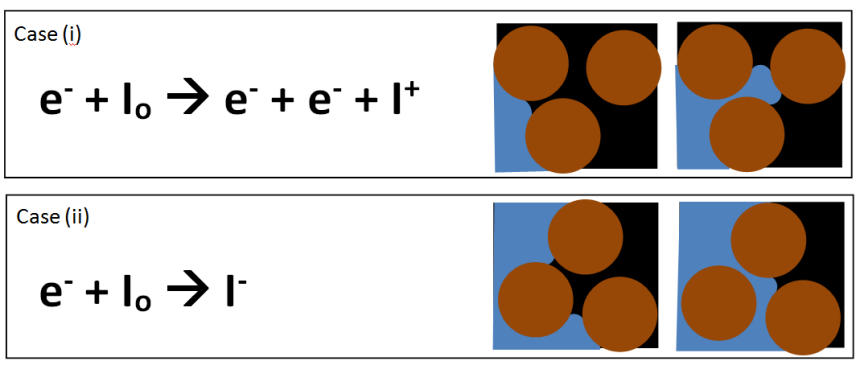

$\mathbf{e}^{-}+\mathbf{I}_{0} \rightarrow \mathbf{e}^{-}+\mathbf{I}_{0}$

FIG. 1. (Color online) (a) Schematic showing the three possibilities of electron propagation during dielectric breakdown as described by [12] and (b) some analogous case for a meniscus movement in a porous medium.

ionization which occurs only above a minimum excitation potential. These electrons move along the field and make collisions with other molecules. These collisions can lead to more electrons (case i: generation) that propagate the discharge further or could result in the annihilation of the electron (case ii: absorption), causing that branch of electric discharge to stop [Fig. 1(a)]. The elastic collision (case iii: elastic collision in DBM), which does not generate or annihilates the electron, is not of great interest in the DBM context. Figure 1(b) shows the similarity to pore-scale flow in porous media during drainage, where a meniscus jumps through one pore throat when the pressure is above the invasion capillary pressure of the throat. This meniscus can make a jump through a pore throat (Haines jump) and then branch into two menisci after crossing the adjacent pore body. This leads to generation of a new meniscus (case i). Two menisci can merge into one meniscus at a pore body, causing a loss of meniscus (case ii). Lastly, a meniscus can make a Haines jump without adding a new meniscus (case iii). Pietronero et al. [12] proposed that the relationship between the local electric field and growth probability for a breakdown is linear $(\eta=1)$ because it depends on the velocity of electrons from one point to the other and the subsequent electron discharge occurs spontaneously. For a porous medium, the flow of the fluid is definitely proportional to the pressure gradient, but each pore throat has a different invasion pressure and adds resistance to the flow. The flow also depends on the viscosities of the fluids and the wettability of the pore walls. Therefore, the relationship is nonlinear. We intend to introduce the effect of finite viscosity ratio through the parameter $\eta$.

For two-phase, immiscible, incompressible displacement in homogeneous porous media, the pressure fields can be written as [18]

$$
\begin{gathered}
\nabla^{2} P_{w}=0 ; \quad P_{w 1}=P_{\text {injection }} \text { and } P_{w 2}=P_{\text {interface }}, \\
\nabla^{2} P_{o}=0 ; \quad P_{o 1}=P_{\text {interface }} \text { and } P_{o 2}=P_{\text {outlet }},
\end{gathered}
$$

where $P_{w}$ is the pressure in the water phase, $P_{\text {injection }}$ is the pressure at the injector, $P_{\text {interface }}$ is the pressure at the oil-water 
interface, $P_{o}$ is the pressure in the oil phase, and $P_{\text {outlet }}$ is the pressure at the outlet. By assuming $P_{w 2}=P_{o 1}=P_{\text {interface }}$ in Eqs. (5) and (6), it is assumed that the capillary force is negligible in comparison to the viscous forces. Since the pressure at the interface is variable and the location of the interface is variable and unknown at each time step, this equation is difficult to solve. For the case of negligible viscosity of the injected water phase, the pressure gradient in the water phase can be neglected and

$$
\nabla^{2} P_{o}=0 ; \quad P_{o 1}=P_{\text {interface }}=P_{\text {injection }} \text { and } P_{o 2}=P_{\text {outlet }} \text {. }
$$

It is evident that Eq. (7) is similar to Eq. (1); i.e., the pressure field in the viscous oil phase is similar to the potential field in the uninvaded region of DBM. A pseudopotential term can be defined $\left(\bar{\phi}_{o}\right)$ as

$$
\bar{\phi}_{o}=\frac{P_{o}(t)-P_{\text {interface }}}{P_{\text {outlet }}-P_{\text {interface }}} .
$$

This converts Eq. (7) to

$$
\nabla^{2} \bar{\phi}_{o}=0 ; \quad \bar{\phi}_{o 1}=0 \text { and } \bar{\phi}_{o 2}=1 .
$$

The boundary conditions $\bar{\phi}_{o 1}=0$ and $\bar{\phi}_{o 1}=0$ are the normalized constant pressure boundary conditions at a particular time step at the injector and producer, respectively.

Keeping in mind all the similarities between two-phase incompressible flow in porous media and dielectric discharge discussed earlier in this section, an extension of DBM is suggested for finite viscosity ratios. The value of the power exponent $\eta$ is changed from 1 to values less than 1 in Eq. (4). Figure 2 sketches the growth probability distribution along the interface as a function of $\mu_{r}$ (or $\eta$ ) for an initial flat front with a single perturbation (assuming that there are 100 points on the interface and the point at the middle is first invaded). The case $\eta=1$ is the base case of the probability distribution along the points on the interface for DBM or infinite viscosity ratio. The distribution has a sharp spike at the center and hence the tip grows faster. As the value of $\eta$ is reduced the curve starts to flatten and the probability distribution is almost uniform for $\eta=$ $10^{-4}$. This suggests that the effects of perturbation have less chance to grow for smaller values of $\eta$ and the displacement is more stable.

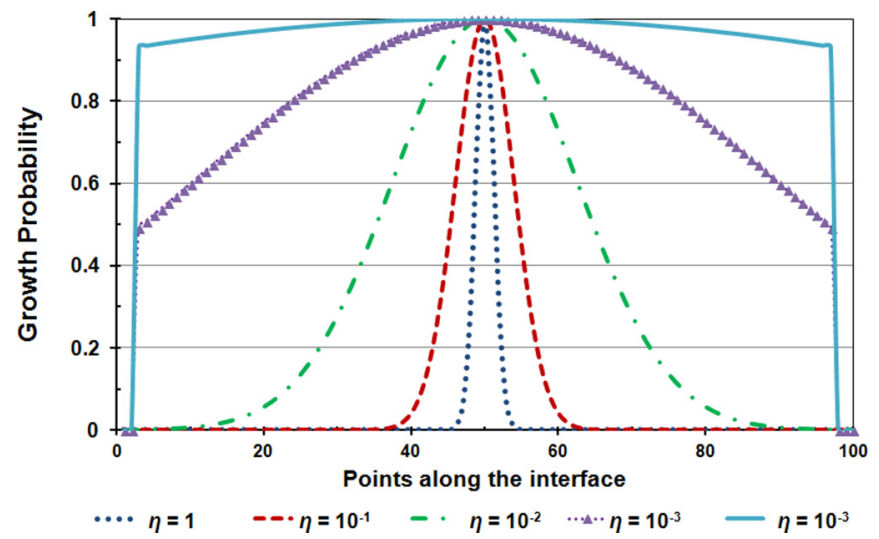

FIG. 2. (Color online) A plot of growth probability distribution along a flat interface with a small perturbation at the center for different values $\eta$ (or $\mu_{r}$ ).
The potential field is calculated by iteratively solving the system of Eq. (9) even in the case of finite viscosity ratio. Thus, the pressure gradient in the injected phase is still neglected to keep the algorithm simple. It is, in a way, similar to the position-space renormalization as suggested by Nagatani and Stanley [9] and Lee et al. [10]. The process is repeated for each grid and iterated until the values do not change beyond a certain assigned value of tolerance $\left(<10^{-4}\right)$. The model is also tested for a smaller value of tolerance $\left(10^{-7}\right)$; this slows down the simulation considerably and does not change the results qualitatively. After the iterations converge and the new potential field is calculated, Eq. (4) is used to calculate the probability for each interface movement. This probability is multiplied with a random number to account for local fluctuations; the movement with the highest probability is executed and the process is repeated again.

As pointed out by Lenormand et al. [19], two-phase, immiscible flow in porous media is influenced by both capillary and viscous effects. It is interesting to note that even though the capillary forces are not explicitly added in the model, the multiplication of a random number implicitly brings in the effect of capillary forces for smaller values of $\eta$ (low viscosity ratios, where capillary effects are important). The small value of power exponent $\eta$ (say $10^{-4}$ ) when applied to the calculated probability based on potential, evens out the differences in growth probability and the invaded grid is selected randomly or based on the pore throat distribution. For example, consider two grids with growth probability of $P_{1}=$ 0.9 and $P_{2}=0.7$ and two cases with $\eta=0.0001$ and $\eta=$ 0.1 . For $\eta=0.0001$ after applying the power exponent $P_{1}=$ 0.9998 and $P_{2}=0.9996$; in this case randomness dictates the invading grid. For $\eta=0.1, P_{1}=0.989$, and $P_{2}=0.964$ in this case, both randomization and potential are important.

Our model does not take into account capillary pressure and pore size distribution explicitly for the sake of simplicity of the model. It assumes a homogeneous permeability and pore size (away from the interface). Near the fingertip, it accounts for the variability of the pore throats through the use of a random number. This model is more suited to drainage processes where there is a positive critical invasion pressure and both viscous forces and capillary forces favor invasion of the larger pores by the injected nonwetting phase. During imbibition, viscous flow favors invasion through bigger pores

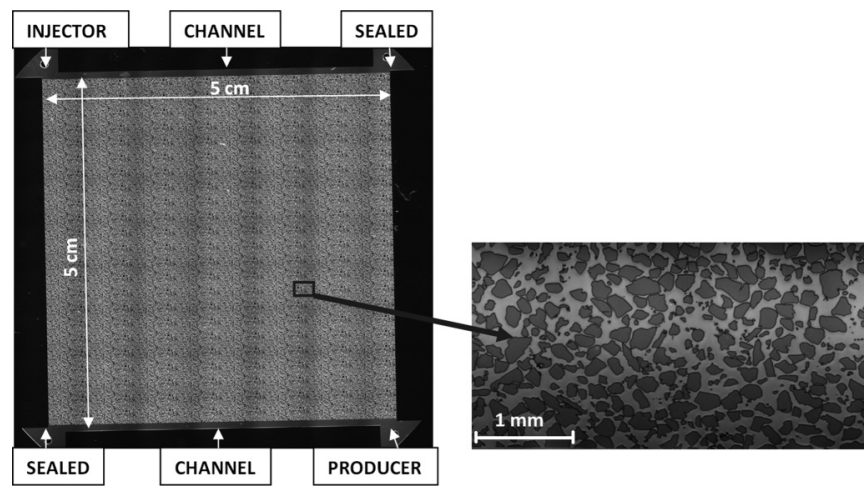

FIG. 3. A picture of the silica micromodel used in the study showing the location of the inlet and outlet ports and an enlarged image of the etched pore-scale pattern. 
TABLE I. List of fluid viscosities, interfacial tensions, oil-silica surface contact angles, and capillary numbers for each experiment in the study.

\begin{tabular}{lcccccc}
\hline \hline & Injected phase & Displaced phase & Interfacial tension & Contact angle & Capillary number $\left(\mu_{w} v / \sigma\right)$ & Capillary number $\left(\mu_{o} v / \sigma\right)$ \\
\hline$\mu_{r}=0.005$ & $1200 \mathrm{cp}$ & $6 \mathrm{cP}$ & $16 \mathrm{dyn} / \mathrm{cm}$ & $110^{\circ}-115^{\circ}$ & $1.25 \times 10^{-3}$ & $6.25 \times 10^{-6}$ \\
$\mu_{r}=1$ & $1 \mathrm{cp}$ (brine) & $1 \mathrm{cP}$ & $20 \mathrm{dyn} / \mathrm{cm}$ & $122^{\circ}-128^{\circ}$ & $8.33 \times 10^{-6}$ & $8.33 \times 10^{-6}$ \\
$\mu_{r}=200$ & $1 \mathrm{cp}$ (brine) & $200 \mathrm{cP}$ & $32 \mathrm{dyn} / \mathrm{cm}$ & $95^{\circ}-100^{\circ}$ & $5.21 \times 10^{-6}$ & $1.04 \times 10^{-3}$ \\
$\mu_{r}=1000$ & $1 \mathrm{cp}$ (brine) & $1000 \mathrm{cP}$ & $32 \mathrm{dyn} / \mathrm{cm}$ & $100^{\circ}-102^{\circ}$ & $5.21 \times 10^{-6}$ & $5.21 \times 10^{-3}$ \\
$\mu_{r}=4000$ & $1 \mathrm{cp}$ (brine) & $4000 \mathrm{cP}$ & $35 \mathrm{dyn} / \mathrm{cm}$ & $90^{\circ}-92^{\circ}$ & $4.76 \times 10^{-6}$ & $1.9 \times 10^{-2}$ \\
$\mu_{r}=10000$ & $1 \mathrm{cp}$ (brine) & $10000 \mathrm{cP}$ & $36 \mathrm{dyn} / \mathrm{cm}$ & - & $4.63 \times 10^{-6}$ & $4.63 \times 10^{-2}$ \\
\hline \hline
\end{tabular}

and capillary pressure favors the smaller pores; the snap-off process is also important. Thus our current model is not applicable to imbibition processes.

\section{EXPERIMENTS}

This section describes the micromodel experimental setup, details of the fluids used in the study, and the results of the experiments conducted.

\section{A. Experimental setup}

The micromodels used in this study were etched silica micromodels with $5 \mathrm{~cm} \times 5 \mathrm{~cm}$ of etched area and a random grain pattern. The etch depth was estimated to be about 25 microns. Figure 3 shows a picture of the micromodel with an enlarged section showing the etched pattern. The silica surface can be classified as slightly oil wet based on the contact angle experiments. Contact angle values between silica and oil phase, measured through the water phase, are listed in Table I. During the experiment, the micromodels were first vacuumed and then saturated with brine. The brine was later displaced with the corresponding oil and the system was left to rest for a day before starting the water flood. All the experiments were conducted at a constant injection rate of $5 \mu \mathrm{l} / \mathrm{min}$. Table I contains the details of the fluids used in the study; all the experiments and measurements were conducted at room temperature of $25^{\circ} \mathrm{C}$. Oil-water interfacial tensions were measured using the pendent-drop apparatus and are also listed in Table I.
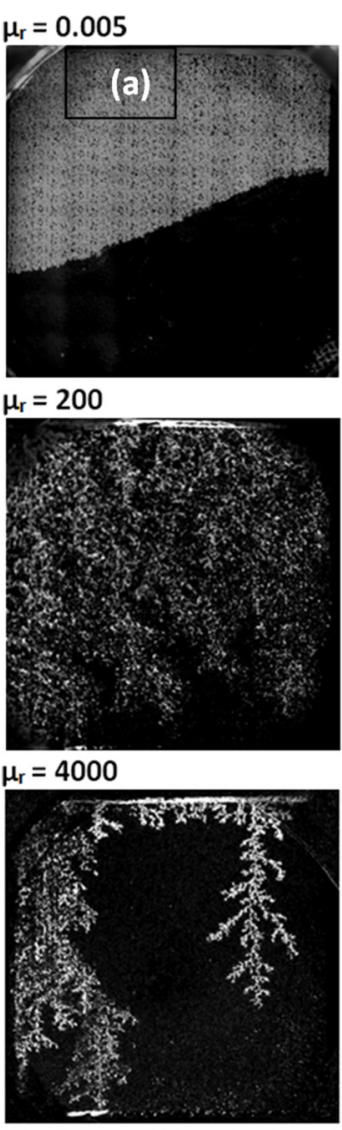

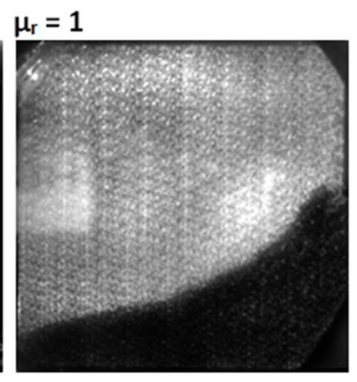

$\mu_{\mathrm{r}}=1000$

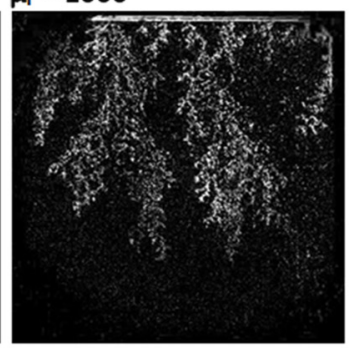

$\mu_{\mathrm{r}}=10,000$

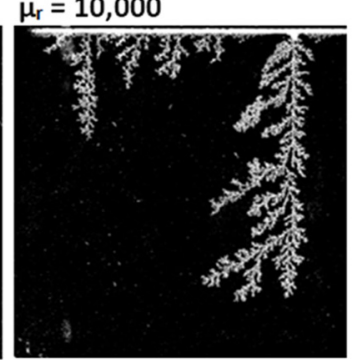

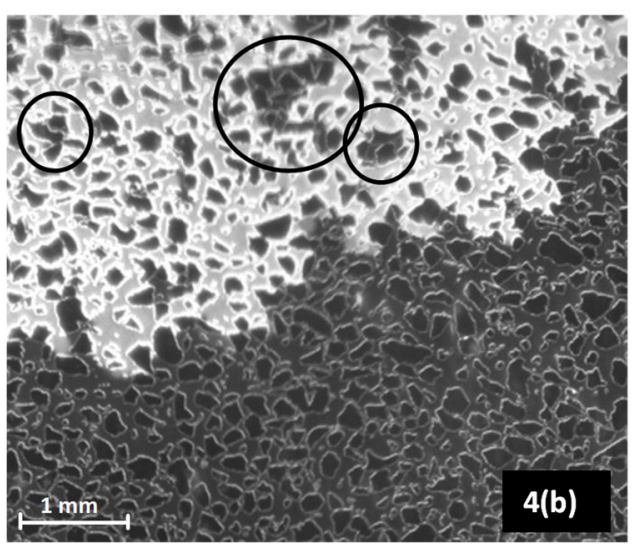

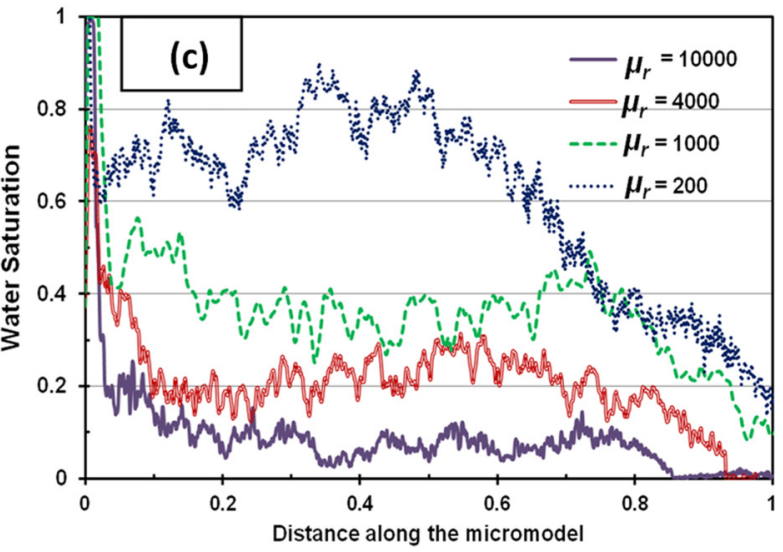

FIG. 4. (Color online) (a) Displacement patterns generated in a 2D silica micromodel while flooding at viscosity ratios of $0.005,1,200$, 1000, 4000, and 10,000; (b) an expanded view of stable displacement with $\mu_{r}=0.005$ showing the trapped oil in the swept zone and the pore-scale perturbations that exist in even the most stable displacement; (c) saturation profile along the length of the micromodel at the time of breakthrough for $\mu_{r}=200$ and above. 

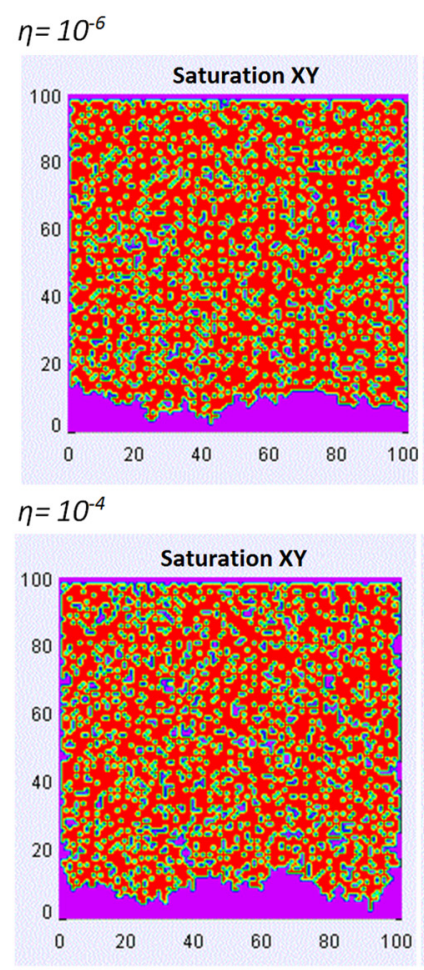

$\eta=0.5 \times 10^{-2}$

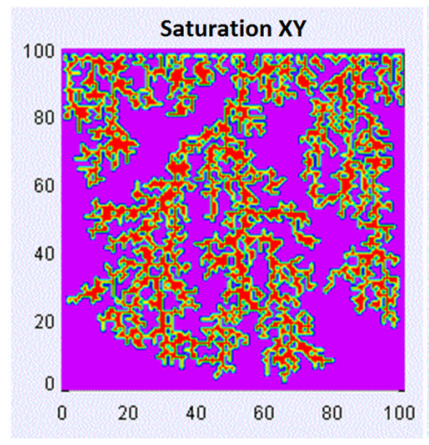

$\eta=0.5 \times 10^{-1}$

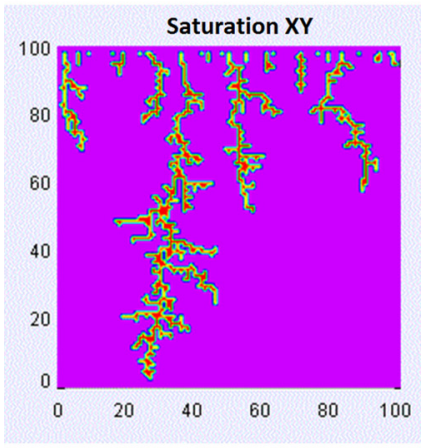

(a) $\eta=10^{-5}$

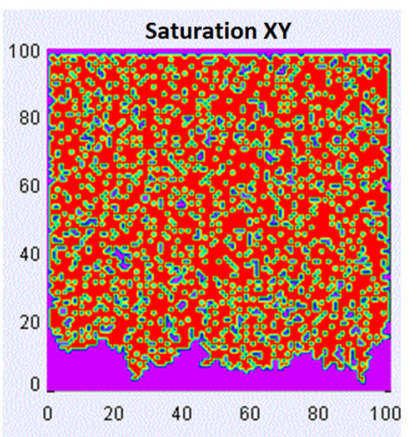

$\eta=10^{-3}$

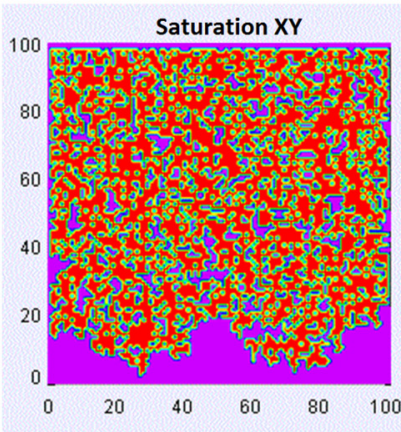

$\eta=10^{-2}$

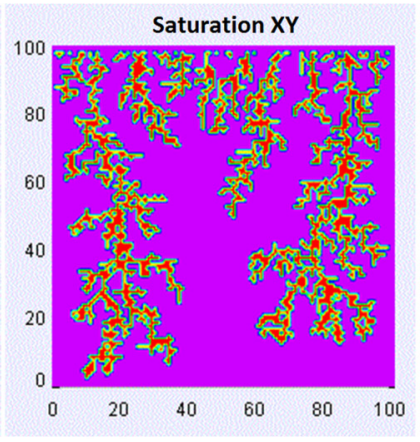

$\eta=10^{-1}$

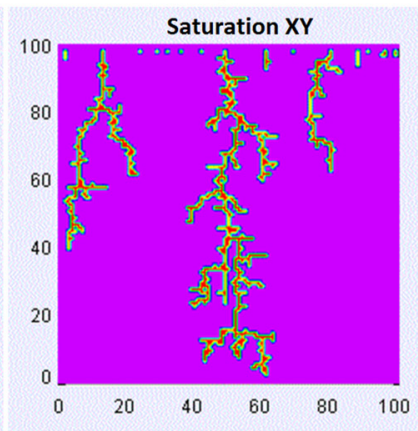

$\eta=10^{-6}$

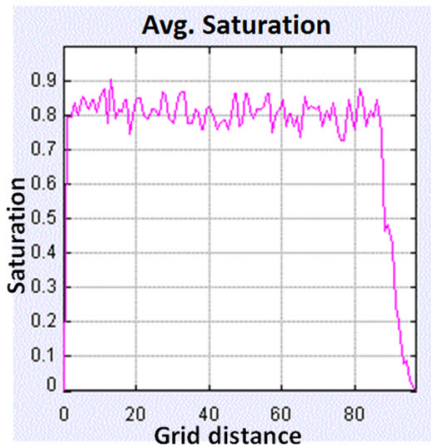

$\eta=10^{-4}$

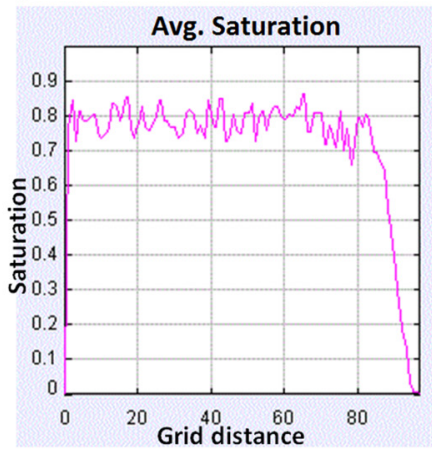

$\eta=0.5 \times 10^{-2}$

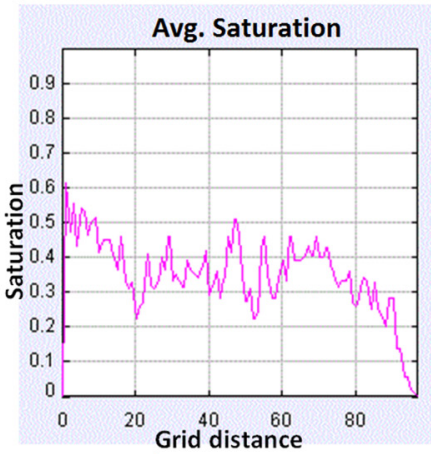

$n=0.5 \times 10^{-1}$

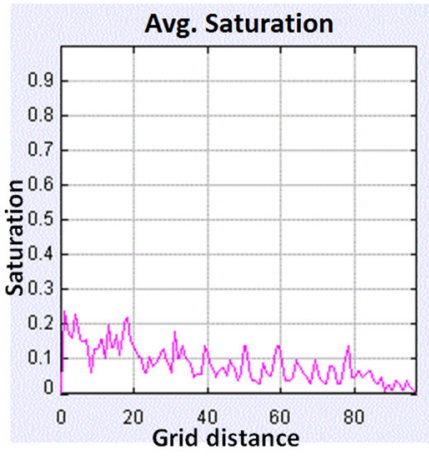

$\eta=10^{-5}$
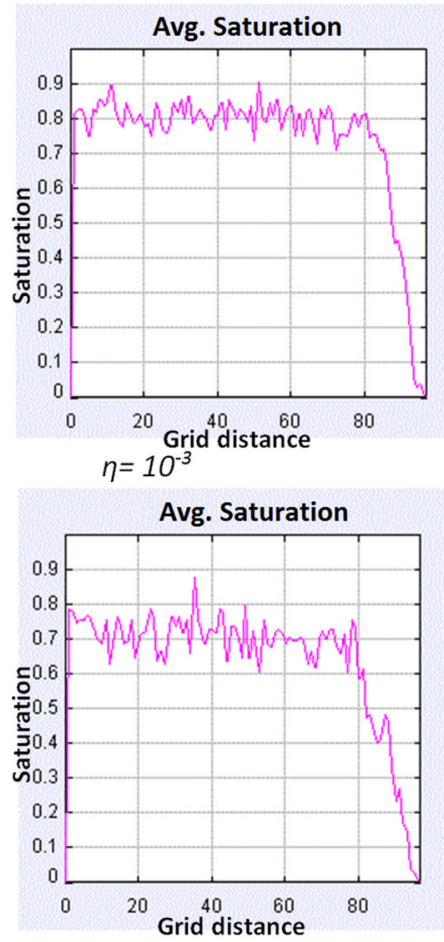

$\eta=10^{-2}$

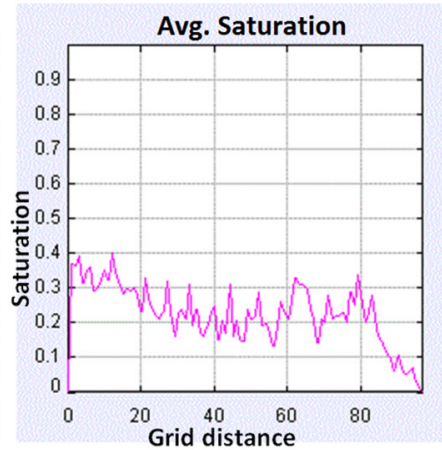

$n=10^{-1}$

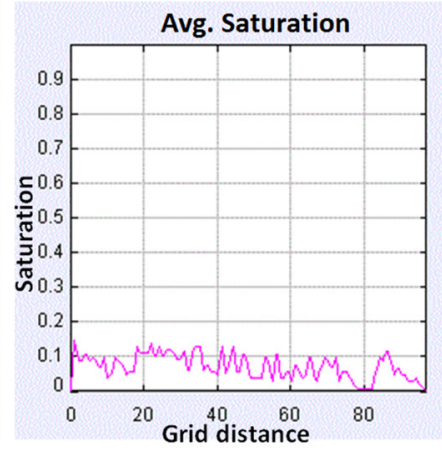

(b)

FIG. 5. (Color online) (a) Water and oil distribution in 2D simulations for different values of parameter $\eta$; (b) plot of water saturation along the length of the matrix at the time of breakthrough. "Saturation $X Y$ " is the phase distribution in the $X Y$ plain and "Avg. Saturation" is the average along the length.

\section{B. Results}

The results of the viscous fingering experiments conducted in a 2D silica micromodel are presented in this section. Figure 4(a) shows the viscous patterns developed in silica micromodels for the viscosity ratios $\left(\mu_{r}\right)$ of $0.005,1,200$, 1000,4000 , and 10000 at an injection rate of $5 \mu \mathrm{l} / \mathrm{min}$. The capillary number based on the displacing phase viscosity, $\mu_{w} v / \sigma$, is about $10^{-6}$, as shown in Table I, except for the first case. The capillary number based on the displaced phase 

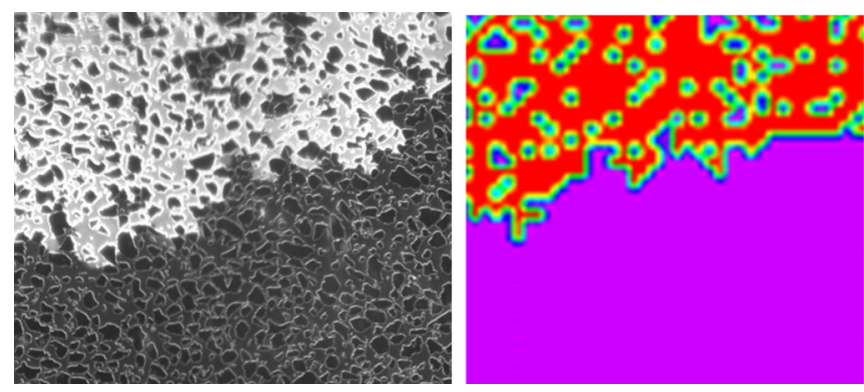

FIG. 6. (Color online) A comparison of the experimental 2D micromodel result and simulation at $\mu_{r}=0.005$ and $\eta=10^{-6}$, respectively. Pink color (lower half) indicates the oil phase; red (top, speckled portion) is the water phase with trapped oil.

viscosity, $\mu_{o} v / \sigma$, corresponding to this flow rate varies from about $10^{-6}$ to $10^{-2}$.

In this study, we observed that the displacement at the viscosity ratio of 0.005 and 1 is stable; the displaced region is compact with few trapped isolated oil blobs. Figure 4(b) shows the close-up version of displacement at $\mu_{r}=0.005$ showing the residual oil blobs in the swept region and the pore-scale perturbations in the stable front. At the viscosity ratio of 200, the invaded region is less efficiently displaced; the fingers are many pores thick. At the viscosity ratio of 1000 , the invaded region is even less efficiently displaced; the fingers are thinner and directed mostly in the flow direction. At the range of viscosity ratio of $4000-10000$, finger width is only a few pores wide, and the displacement pattern is like diffusion limited aggregation (DLA). Also the separation in between the fingers increases as the viscosity ratio increases; for $\mu_{r}=$ 10000 there is only one prominent finger growing, for $\mu_{r}=$ 4000 there are two contributing fingers, and for 200 and 1000 there are multiple fingers that lie close to each other. Figure 4(c) shows the saturation profiles along the length of the micromodel for each case.

\section{SIMULATIONS}

To match our experimental setup, the model was applied to a rectangular domain. A flow potential of $\phi_{w}$ is specified

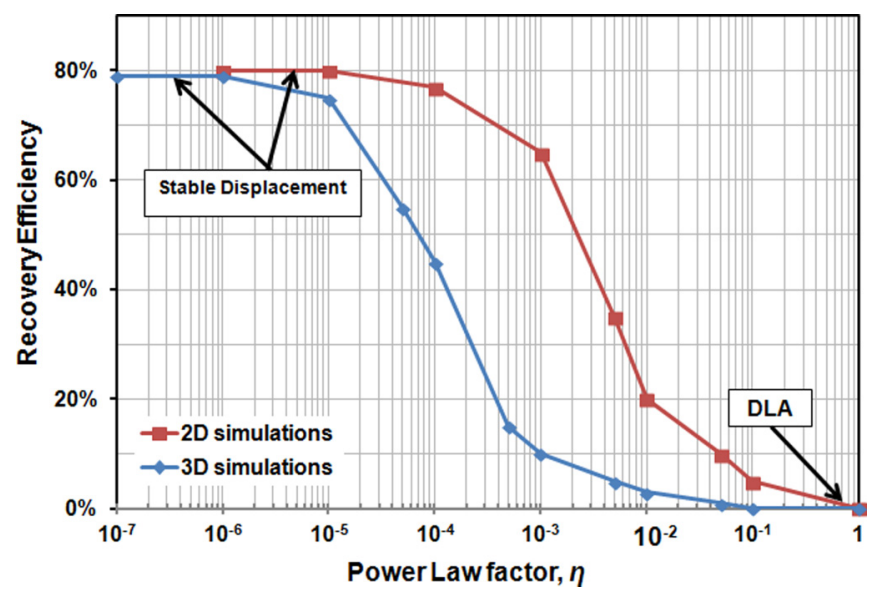

FIG. 7. (Color online) A plot of recovery efficiency vs power-law factor $(\eta)$ for $2 \mathrm{D}$ and $3 \mathrm{D}$ simulations.
TABLE II. Recovery efficiency and viscosity ratio data from the experiment along with the matching value of $\eta$.

\begin{tabular}{lcl}
\hline \hline Viscosity ratio, $\mu_{r}$ & Recovery efficiency & \multicolumn{1}{c}{$\eta$} \\
\hline 1 & 0.8 & 0.00001 \\
200 & 0.65 & 0.001 \\
1000 & 0.35 & 0.005 \\
4000 & 0.2 & 0.01 \\
10000 & 0.08 & 0.04 \\
\hline \hline
\end{tabular}

for all the sites at the inlet. The sites at the producer are assigned a flow potential of $\phi_{0}$. The grids between the inlet and the outlet can be assigned some random values in the beginning and their actual values are computed while solving the Laplace equation. Each grid (or site) represents a pore body and each connection between grids (or bond) represents a pore throat connecting pore bodies. Pore volume is associated with pore bodies and flow resistance is assigned to pore throats.

\section{A. 2D simulations}

For this study, a $100 \times 100 \times 1$ grid system was chosen to represent our 2D micromodel system. We focus on demonstrating that our model can produce displacement patterns similar to the experimental ones for different values of $\eta$, which correspond to different viscosity ratios. The results of the 2D network model simulations are shown in Figs. 5(a) and 5(b). Later, a graphical correlation between $\eta$ and viscosity ratio is developed by matching the recovery efficiency of the micromodel experiments and 2D simulations.

It is interesting to note that the simulation captures most of the features of the micromodel experiments (which are 2D). There is always some remaining oil in the swept zones, even for the smallest value of $\eta$ (most stable case) and the interface has pore-scale perturbations that lead to this residual. Figure 6 shows a comparison of the expanded sections of a stable micromodel displacement at $\mu_{r}=0.005$ and simulations at $\eta=10^{-6}$. As the viscosity ratio is increased the remaining oil saturation and the separation in between the fingers increase. At $\eta$ between $10^{-6}$ and $10^{-4}$ (low viscosity ratio), the

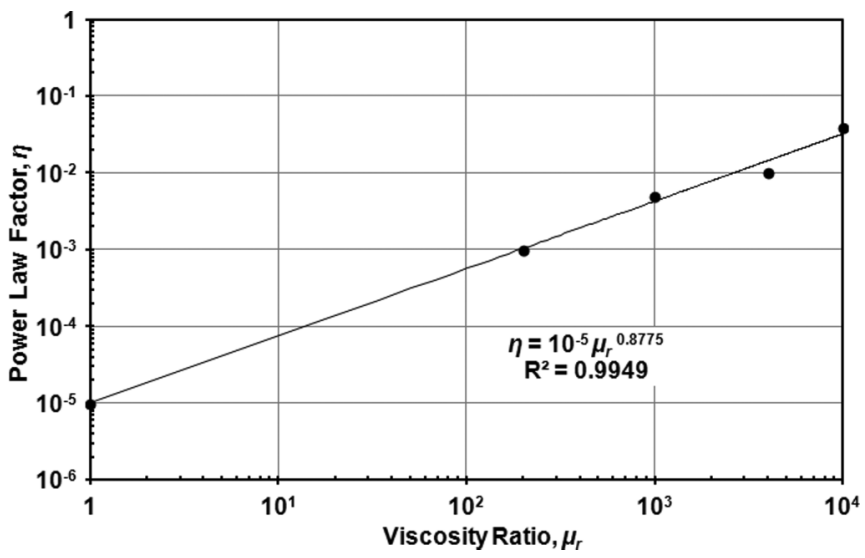

FIG. 8. Relation between $\eta$ and viscosity ratios based on the 2D micromodel experimental results. 

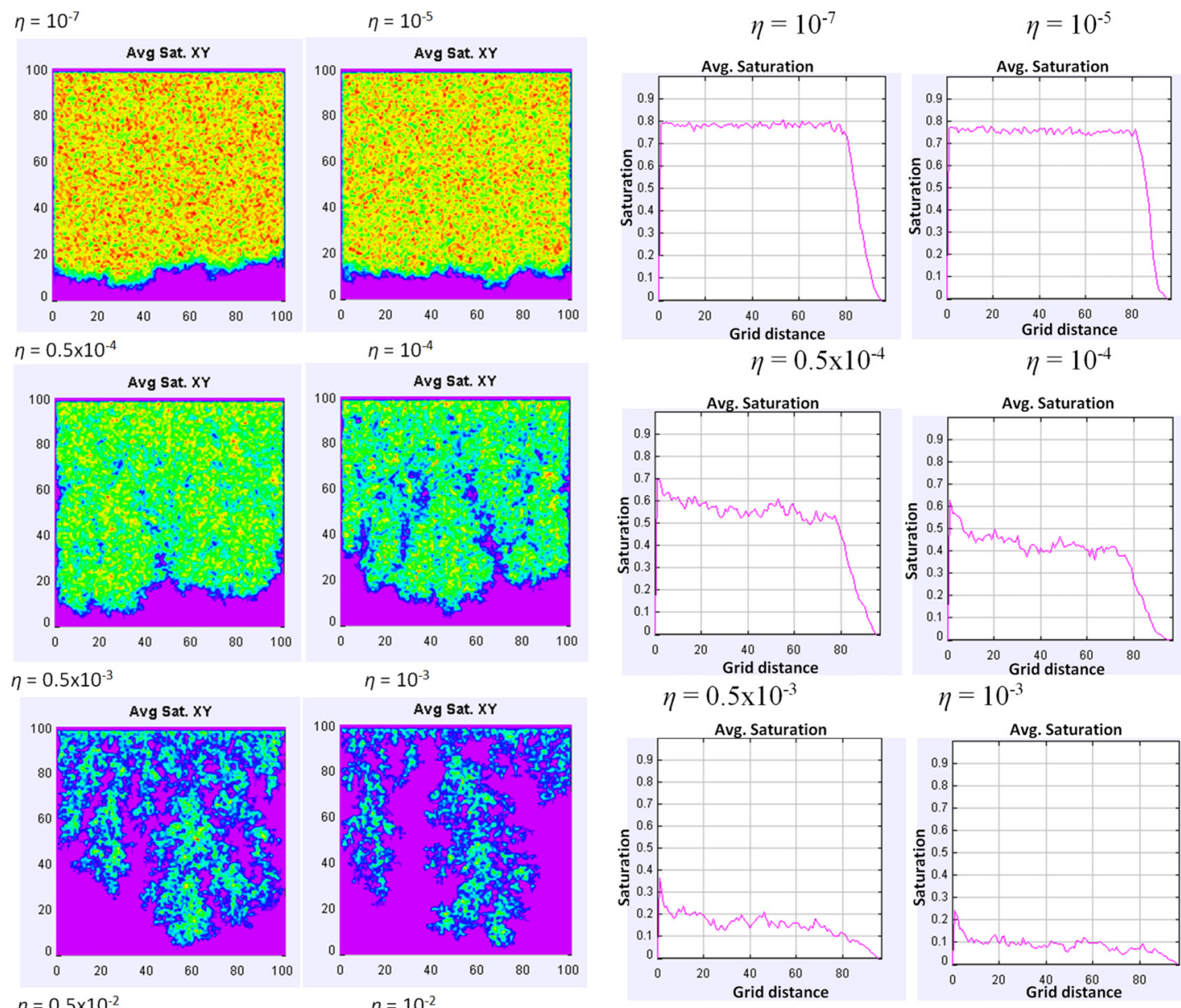

$$
\eta=0.5 \times 10^{-3}
$$

$\eta=10^{-3}$
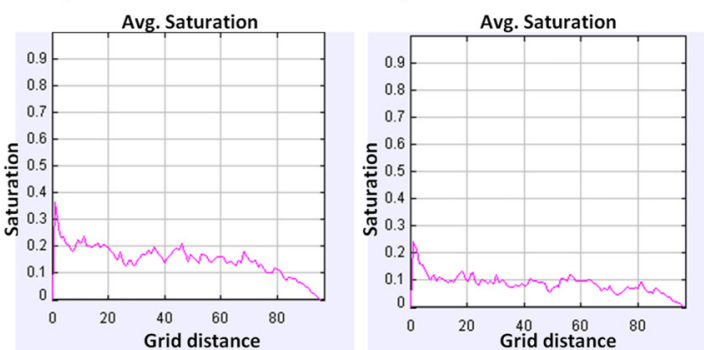

$$
n=0.5 \times 10^{-2}
$$
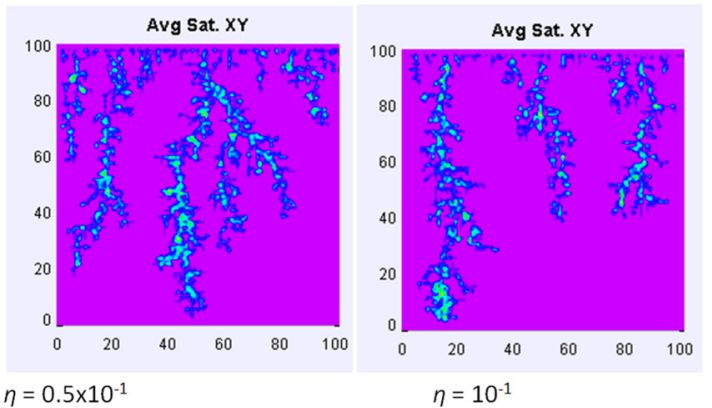

$$
\eta=0.5 \times 10^{-2}
$$

$\eta=10^{-2}$
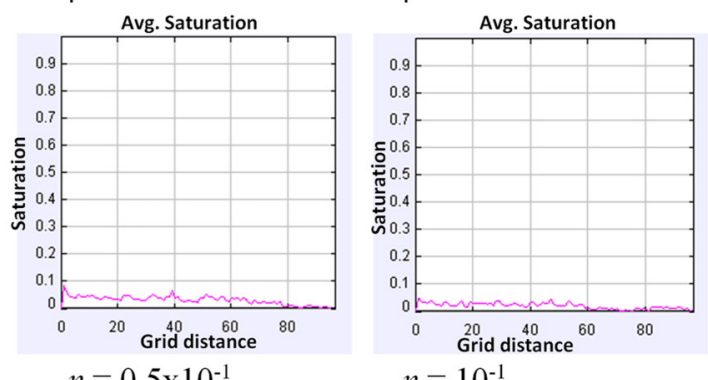

$$
\eta=0.5 \times 10^{-1}
$$
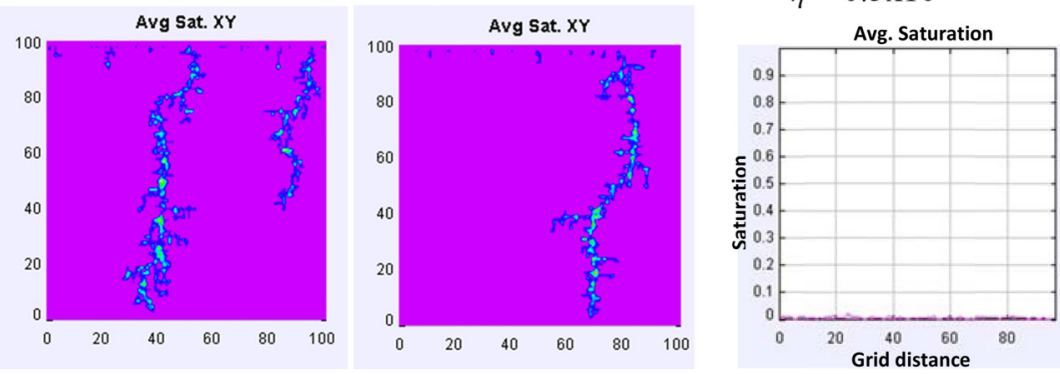

$\eta=10^{-1}$

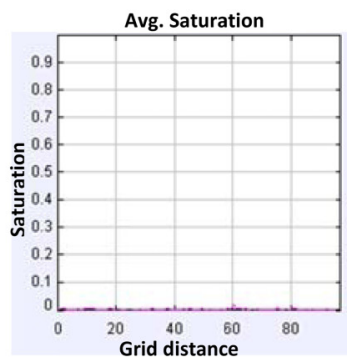

(b)

FIG. 9. (Color online) (a) Averaged water and oil distribution in the $X Y$ plain (Avg. Sat $X Y$ ) for 3D simulation with different values of parameter $\eta$; (b) plot of water saturation along the length of the matrix at the time of breakthrough.

displacement is stable and the swept area has a displacement efficiency of $80 \%$ ( $20 \%$ remaining). As the value of $\eta$ is increased, the stable front starts to break into thick fingers and the remaining oil saturation $\left(S_{\text {or }}\right)$ in the swept area increases. On further increase of $\eta$, thin fingers that start to resemble DLAlike fractals appear. Figure 7 presents a plot of recovery efficiency $\left(1-S_{\text {or }}\right)$ vs $\eta$ for the $2 \mathrm{D}$ simulations. For smaller values of $\eta\left(\eta<10^{-4}\right)$ all patterns converge into a stable displacement 
and for values close to $1(\eta \sim 1)$ the pattern approaches the DLA model.

Table II shows the recovery efficiency in micromodel experiments as a function of viscosity ratio. As the viscosity ratio increases, the recovery efficiency decreases. Table II also denotes the parameter $\eta$ for which a similar displacement efficiency was observed in 2D simulations. Figure 8 shows the relation between $\eta$ and viscosity ratio $\left(\mu_{r}\right)$ for $2 \mathrm{D}$ simulations listed in Table II. The correlation between parameter $\eta$ and $\mu_{r}$ can be expressed as

$$
\eta=10^{-5} \mu_{r}^{0.8775} .
$$

Sherwood and Nittmann [14] presented a similar model without the use of the exponent $\eta$. Their stable displacement had perturbations at the interface, but the sweep in the invaded region was always $100 \%$. In our model, the recovery efficiency never goes beyond $80 \%$ for all finite values of $\eta$. Randomness causes menisci to isolate pores with oil and cause capillary trapping of the oil phase. Since the sites occupied by the invading phase are assigned $\phi=0$ and the pressure field in the invading phase is considered negligible, an isolated blob is surrounded by grids that are assigned zero potential and is disconnected from the $\phi=1$ boundary condition at the outlet. When the Laplacian is solved iteratively for sites on the isolated island, the potential becomes zero and therefore, for all finite values of $\eta$, once isolated any oil blob cannot be displaced. Only for $\eta=0$, the probability of these blobs along with every point on the interface is $1\left(0^{\circ}=1\right)$; so the islands are still created, but they disappear with time.

\section{B. 3D simulations}

Having tested the model in 2D, simulations were conducted on a $100 \times 100 \times 15$ grid system and Eq. (3) was used for solving the Laplacian for a 3D system. Recently Bondino et al. [20] and Skauge et al. [21] have published results of X-ray scans of viscous fingers in a 12 in. $\times 12$ in. $\times 1$ in. Bentheimer slab. These images were used as a qualitative reference for our simulations. Figures 9(a) and 9(b) show the results of the simulation for the 3D domain.

The trend observed in 3D simulations is similar to that observed in 2D simulations. The system evolves as a stable displacement at low values of $\eta$ and as the value of $\eta$ is

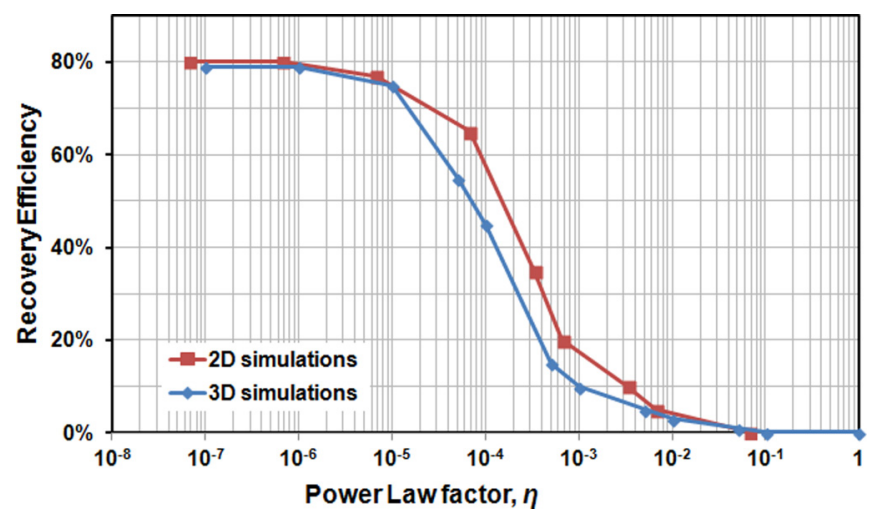

FIG. 10. (Color online) A plot showing that the recovery trends of $2 \mathrm{D}$ and $3 \mathrm{D}$ simulations are scalable.
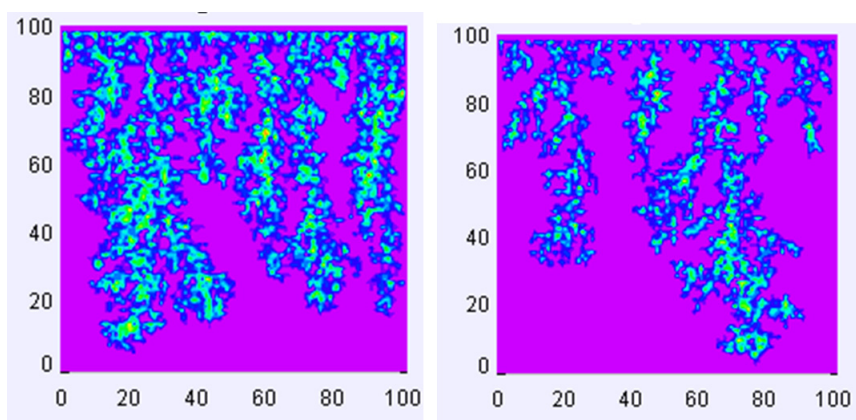

FIG. 11. (Color online) Simulations of oil displacement for $\eta=$ $3.16 \times 10^{-3}$ and $1.05 \times 10^{-3}$ to simulate the 2000 and 7000 viscosity ratio displacement experiments in Bentheimer slabs presented by Skauge et al. [21].

increased to unity, the system transforms from thick fingers into DLA-like fractal fingers. For all the finite values of $\eta$ (up to $10^{-7}$ ), the recovery efficiency is below $80 \%$. This is consistent with the experiments and our $2 \mathrm{D}$ simulations, where $100 \%$ recovery is never achieved in porous media for immiscible displacements because of capillary trapping. In Fig. 7, recovery efficiency is plotted vs power-law index $\eta$ for $3 \mathrm{D}$ simulations along with $2 \mathrm{D}$ results. It is also interesting to note that the $\eta$ values for the same recovery efficiency in 3D and 2D simulations differ by a factor of approximately 15 which is also the number of grids in the $z$ direction. Figure 10 shows the two curves of Fig. 7 after the 2D $\eta$ values have been scaled down by a factor of 15 . This suggests that the correlation between $\eta$ and $\mu_{r}$, obtained from 2D analysis, can also be scaled up for 3D. By this logic the new correlation for the $3 \mathrm{D}$ systems is

$$
\eta=6.67 \times 10^{-7} \mu_{r}^{0.8775} .
$$

Figures 6 and 7 of Skauge et al. [21] show the x-ray scans of water injection in Bentheimer slabs originally saturated with oils of viscosity 7000 and $2000 \mathrm{cP}$. The slabs used in this study were about $12 \times 12 \times 1$ in. in dimension and therefore a $100 \times 100 \times 8$ gridding scheme was chosen for the simulation to maintain the $12: 1$ aspect ratio. The boundary conditions were similar to the experiments. Details about the spatial distribution of porosity and permeability were not provided in [21] and they were not modeled. However, a Bentheimer rock is known to be quite homogeneous. In our simulation, we do not assign any pore size distribution explicitly. The value of $\eta$ for viscosity ratios of 2000 and 7000 can be calculated to be 3.16 $\times 10^{-3}$ and $1.05 \times 10^{-3}$ for 2000 and $7000 \mathrm{cP}$ oil, respectively. The results of the simulation in $3 \mathrm{D}$ for the two cases are shown in Fig. 11 and are qualitatively very similar to Figs. 6 and 7 of Skauge et al. [21]. More experimental data in 3D are needed to validate and fine-tune the correlation for 3D systems.

\section{CONCLUSIONS}

Based on the similarities between dielectric breakdown and flow in porous medium, an extension of the existing DBM model has been proposed to predict viscous fingering patterns at finite viscosity ratios. The model is seen to be qualitatively consistent with the fingers observed in our $2 \mathrm{D}$ 
micromodel experiments and also with the published results for a larger 3D slab. Smaller values of the power-law index $\eta$ produce a stable displacement front while values close to unity converge to DLA-like fractals. Lastly, a correlation between $\eta$ and the viscosity ratio is developed for the $2 \mathrm{D}$ system by comparing simulations and micromodel experiments, i.e., $\eta=$ $10^{-5} \mu_{r}{ }^{0.8775}$. The 2D and 3D simulation data appear scalable. The fingering pattern in 3D simulation at finite viscosity ratios appears qualitatively similar to the few experimental results published in the literature.
[1] R. Lenormand, Proc. R. Soc. London, Ser. A 423, 159 (1989).

[2] L. Cueto-Felgueroso and R. Juanes, Phys. Rev. Lett. 101, 244504 (2008).

[3] D. S. Hughes and P. Murphy (unpublished).

[4] D. S. Hughes and P. Murphy, SPE Reservoir Eng. 3, 1129 (1988).

[5] T. A. Witten and L. M. Sander, Phys. Rev. B 27, 5686 (1983).

[6] L. Paterson, Phys. Rev. Lett. 52, 1621 (1984).

[7] P. Meakin, Phys. Rev. A 27, 2616 (1983).

[8] T. Nagatani, Phys. Rev. A 40, 7286 (1989).

[9] T. Nagatani and H. E. Stanley, Phys. Rev. A 41, 3263 (1990).

[10] J. Lee, A. Coniglio, and H. E. Stanley, Phys. Rev. A 41, 4589(R) (1990).

[11] L. Niemeyer, L. Pietronero, and H. J. Wiesmann, Phys. Rev. Lett. 52, 1033 (1984).

[12] L. Pietronero and H. J. Wiesmann, Z. Phys. B: Condens. Matter 70, 87 (1988).

[13] K. J. Maaløy, J. Feder, and T. Jøssang, Phys. Rev. Lett. 55, 2688 (1985).
[14] J. D. Sherwood and J. Nittmann, J. Phys. 47, 15 (1986).

[15] T. Clemens, K. Tsikouris, M. Buchgraber, L. Castanier, and A. Kovscek, SPE Reservoir Eval. Eng. 16, 144 (2013).

[16] M. Eden, in Proceedings of the Fourth Berkeley Symposium on Mathematical Statistics and Probability, Vol. 4 (University of California Press, Berkeley, CA, 1961), pp. 223-239.

[17] S. Doorwar and K. K. Mohanty, in Proceedings of SPE Annual Technical Conference and Exhibition, Denver, CO (Society of Petroleum Engineers, Colorado, 2011).

[18] L. W. Lake, Enhanced Oil Recovery (Prentice Hall, Englewood Cliffs, NJ, 1989).

[19] R. Lenormand, E. Touboul, and C. Zarcone, J. Fluid Mech. 189, 165 (1988).

[20] I. Bondino, R. Nguyen, G. Hamon, P. A. Ormehaug, A. Skauge, and S. Jouenne, in Proceedings of the 25th International Symposium of the Society of Core Analysts, Austin, TX (Society of Core Analyst, Dublin, CA, USA, 2011).

[21] A. Skauge, P. A. Ormehaug, T. Gurholt, B. Vik, I. Bondino, and G. Hamon, in Proceedings of SPE Improved Oil Recovery Symposium, Tulsa, OK (Society of Petroleum Engineers, Oklahoma, 2012). 